\title{
Efeitos de doses crescentes de calcário em solo Latossolo Amarelo na produção de mudas de pau-de- balsa (Ochroma lagopus sw., bombacaceae)
}

\author{
Carlos Alberto Franco TUCCI ${ }^{1}$, Hedinaldo Narciso LIMA², Aildo da Silva GAMA3 , Heron Salazar COSTA \\ Patricia Aparecida de SOUZA 5
}

\section{RESUMO}

A produção de mudas de qualidade com adequado teor nutricional é fundamental para o desenvolvimento da planta e para a formação do sistema radicular, a qual apresentará melhor capacidade de adaptação ao novo local após o plantio. O objetivo deste trabalho foi avaliar o efeito de níveis crescentes de calcário na produção de mudas de pau-de-balsa. Os tratamentos foram constituídos de doses crescentes de corretivo e equivaleram a 0,$0 ; 0,25 ; 0,5 ; 0,75 ; 1,0 ; 1,5$ e 2,0 t ha ${ }^{-1}$ de calcário e o delineamento experimental utilizado foi o de blocos casualizados com cinco repetiçóes. As características avaliadas foram: altura da planta; diâmetro do colo, matéria seca da parte aérea, matéria seca total, relaçáo raiz/parte aérea, teores totais de macronutrientes nas plantas ( $\mathrm{N}, \mathrm{P}, \mathrm{K}, \mathrm{Ca}, \mathrm{Mg}$ e $\mathrm{S})$. Os resultados demonstraram que a prática de calagem como fator de correção do solo usado no substrato favoreceu todas as características de crescimento avaliadas na produção de mudas de pau-de-balsa. A correçáo do solo influenciou positivamente a absorção de $\mathrm{Ca}, \mathrm{Mg}$ e $\mathrm{S}$, por outro lado, não apresentou efeitos estatisticamente significativos para a absorção de N, P e K.

PALAVRAS-CHAVE: espécie florestal, teor nutricional, substrato.

\section{Effects of the rising heat in Yellow Oxisoil in the production of silent wood rafts (Ochroma lagopus sw., bombacaceae)}

\begin{abstract}
The production of quality rafts with appropriate nutritional tenor is fundamental for plant development and for forming of root systems, which present best ability to adapt to new locations after plantation. The goal of this work was to evaluate the effect of rising heat levels in the production of silent wood rafts. The treatment was constituted by rising doses of correction and was equal to $0.0 ; 0.25 ; 0.5 ; 0.75 ; 1.0 ; 1.5$, and $2.0 \mathrm{t} \mathrm{ha}^{-1}$ of lime and the experimental detail used was of blocks, repeating five times. The characteristics evaluated were: plant height; diameter, dry material of the aerial part, root relation/aerial part, content of macronutrients in the plants $(\mathrm{N}, \mathrm{P}, \mathrm{K}, \mathrm{Ca}, \mathrm{Mg}$ and $\mathrm{S})$. The results demonstrate that the practice of liming as a soil correction factor used in the soil substrate improved all the growth characteristics evaluated in seedling production of pau-debalsa (Ochroma lagopus sw., bombacaceae). The soil correction positively influenced the absorption of $\mathrm{Ca}, \mathrm{Mg}$, and $\mathrm{S}$, on the other hand, there weren't any statistically significant effects for absorption of $\mathrm{N}, \mathrm{P}$, and $\mathrm{K}$ resented.
\end{abstract}

KEYWORDS: forest species, nutritional tenor, substrate.

\footnotetext{
1 Universidade Federal do Amazonas, E-mail: ctucci@ufam.edu.br

2 Universidade Federal do Amazonas, E-mail: hedinaldo@ufam.edu.br

${ }^{3}$ Instituto Federal de Educação, Ciência e Tecnologia do Amazonas, Email: aildogama@ifam.edu.br

${ }^{4}$ Universidade Federal do Amazonas, E-mail: hescosta@ufam.edu.br

${ }^{5}$ Universidade Federal do Tocantins, E-mail: patriciaap@hotmail.com
} 


\section{INTRODUÇÃO}

O pau-de-balsa (Ochroma lagopus Sw.) é uma espécie característica das primeiras etapas de sucessão secundária em zonas tropicais úmidas, em temperaturas altas, provocadas pelo calor seco e úmido, produzem aumento de percentagem de germinação das sementes (Vasquez-Yanes, 1974). Ochroma lagopus ocorre na faixa de $19^{\circ} \mathrm{N} \mathrm{e} 20^{\circ} \mathrm{S}$ em regióes tropicais das Américas do Sul e Central e em altitudes de 0 a $1000 \mathrm{~m}$. A madeira é muito leve $\left(0,20 \mathrm{a} 0,35 \mathrm{~g} \mathrm{~cm}^{-1}\right)$ e pode ser utilizados para fabricação de brinquedos, forros de teto, material isolante, para caixa de embalagens, construção aeronáutica e material flutuante (Loureiro et al. 1979), apresentando um alto potencial de uso na fabricação de chapas de cimento-madeira para divisórias, móveis, pisos, etc., com madeira de plantas com cerca de um ano de idade (Barbosa et al. 2004).

A exploração intensiva da floresta amazônica tem ocasionado a perda de recursos florestais valiosos e contribuído para a redução da base genética de inúmeras espécies (Ramos et al. 2006).

A necessidade de produçáo de mudas com melhor qualidade e menor custo em escala comercial é um desafio constante, resultado da crescente demanda de produtos florestais visando o estabelecimento de florestas, povoamentos e para fins industriais. Em função disso, tem-se observado um crescente interesse por parte de agricultores e viveiristas sobre as técnicas de manejo mais adequadas para produçáo de mudas florestais.

A produção de mudas com adequado teor nutricional pressupóem um desenvolvimento e uma boa formaçáo do sistema radicular, com melhor capacidade de adaptaçáo ao novo local após o plantio, apresentando vantagens como aumento da resistência à baixas temperaturas (Carneiro 1995). Portanto, vários trabalhos de pesquisa têm procurado definir o melhor substrato e os níveis de fertilidade do solo para produção de mudas de qualidade de espécies florestais. Resultados positivos a aplicação de corretivo ao substrato na formação de mudas de espécies florestais nativas já foram observados por Tucci et al. (2004) e Silva et al. (2008).

O objetivo deste trabalho foi avaliar os efeitos de diferentes doses de calcário na produçáo de mudas de pau-de-balsa.

\section{MATERIAL E MÉTODOS}

$\mathrm{O}$ experimento foi conduzido em casa-de-vegetação $\mathrm{e}$ em laboratórios da Faculdade de Ciências Agrárias (FCA) da Universidade Federal do Amazonas (UFAM), Manaus, no período de dezembro de 2003 a novembro de 2005. O substrato para a formação de mudas e análise físico-química foi coletado da camada sub-superficial de um Latossolo Amarelo, sob uma cobertura vegetal de uma floresta secundária com mais de 30 anos, localizado no setor sul do campus universitário da UFAM, nas coordenadas geográficas $03^{\circ} 06^{\prime}$ 04 " de latitude sul e 59 58' 34" de latitude oeste.

Para a produçáo de mudas, as sementes de pau-de-balsa foram colocadas para germinar em sementeira, utilizando-se como substrato areia lavada. Após a germinação, quando as plântulas atingiram $12 \mathrm{~cm}$ de altura, foi efetuado uma seleção para homogeneizar quanto ao tamanho e em seguida foi realizado a repicagem.

Os tratamentos foram constituídos de doses crescentes de corretivo e equivaleram a 0,$0 ; 0,25 ; 0,5 ; 0,75 ; 1,0 ; 1,5$ e 2,0 $\mathrm{t}$ ha ${ }^{-1}$ de calcário dolomítico. O delineamento experimental utilizado foi o de blocos casualizados com cinco repetiçóes, num total de 35 parcelas, sendo cada parcela constituída de 4 (quatro) plantas.

O calcário dolomitico foi preparado a partir de uma mistura de $\mathrm{CaCO}_{3}$ (p.a.) e $\left(\mathrm{MgCO}_{3}\right)_{4} \mathrm{Mg}(\mathrm{OH})_{2} 5 \mathrm{H}_{2} \mathrm{O}$ (p.a.), em proporçôes equivalentes e com $100 \%$ de PRNT. Nos tratamentos, a correçáo da acidez foi realizada com trinta dias de antecedência, para que ocorresse a reaçáo de neutralizaçáo da acidez. Após a mistura do terriço com os respectivos tratamentos foi obtido o substrato, em seguida foram acondicionados em sacos plásticos com capacidade de $3,0 \mathrm{~kg}$. O teor de umidade foi mantido constante com o peso da água em $30 \%$ do peso do substrato.

Para corrigir a fertilidade do substrato, em todas as parcelas e depois do período de incubaçáo, o mesmo foi novamente seco, destorroado e peneirado. Daí procedeu-se a fertilização com a utilização de ácido fosfórico $\left(\mathrm{H}_{3} \mathrm{PO}_{4}\right)$, cloreto de potássio $(\mathrm{KCl})$ e sulfato de potássio $\left(\mathrm{K}_{2} \mathrm{SO}_{4}\right)$, em doses equivalentes a 250,150 e $15 \mathrm{~kg} \mathrm{ha}^{-1}$ de $\mathrm{P}_{2} \mathrm{O}_{5}, \mathrm{~K}_{2} \mathrm{O}$ e $\mathrm{S}$. As fontes de $\mathrm{P}, \mathrm{K}$ e $\mathrm{S}$ foram aplicadas na forma de soluçáo e misturadas ao substrato. Também foram aplicadas solução nutritivas de micronutrientes, correspondente a $0,4 \mathrm{mg} \mathrm{kg}^{-1}$ de boro, $0,5 \mathrm{mg} \mathrm{kg}^{-1}$ de cobre, $2,5 \mathrm{mg} \mathrm{kg}^{-1}$ de manganês, 2,5 $\mathrm{mg} \mathrm{kg}^{-1}$ de zinco, $0,05 \mathrm{mg} \mathrm{kg}^{-1}$ de molibdênio e $4,0 \mathrm{mg} \mathrm{kg}^{-1}$ de ferro. As doses foram extrapoladas a partir das sugestôes para eucalipto (Barros 2001). Aos 15, 45 e 60 dias depois da repicagem das plântulas realizou-se adubação com $\mathrm{N}$ em cobertura, utilizando-se uréia $\left(\mathrm{CO}\left(\mathrm{NH}_{3}\right)_{2}\right)$, em dose equivalente a $100 \mathrm{~kg} \mathrm{ha}^{-1}$ de $\mathrm{N}$, aplicada na forma de solução em três parcelas, 40,30 e $30 \mathrm{~kg}$ de $\mathrm{N} \mathrm{ha}^{-1}$.

As características avaliadas foram: altura da planta, medida da base até a gema apical; diâmetro do colo (DC), matéria seca da parte aérea (MSPA), matéria seca de raiz (MSR), matéria seca total (MST) e relação raíz/parte aérea (R/PA).

Foram avaliados os teores totais de macronutrientes nas plantas ( $\mathrm{N}, \mathrm{P}, \mathrm{K}, \mathrm{Ca}, \mathrm{Mg}$ e S), conforme procedimentos metodológicos descritos por Malavolta et al. (1989), no CPAA/EMBRAPA. Os resultados foram convertidos a conteúdos. 
Os dados foram submetidos à análise de variância e as médias foram comparadas pelo teste de Tukey a 5\% de probabilidade.

\section{RESULTADOS E DISCUSSÃO}

\section{CARACTERÍSTICAS QUIIMICAS DOS SUBSTRATOS}

Na Tabela 1, estão expressos os resultados da caracterização do substrato após a aplicação dos tratamentos, onde se pode observar decréscimo no teor $\mathrm{Al}^{3+}$ trocável, com efeito significativo a partir da dosagem de $0,75 \mathrm{t} \mathrm{ha}^{-1}$. Por outro, o

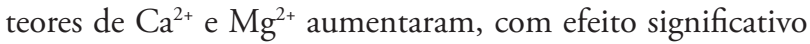
para o $\mathrm{Ca}^{2+}$ a partir da dosagem de $0,75 \mathrm{tha}^{-1}$ e para o $\mathrm{Mg}^{2+}$ houve uma variação de 0,56 a $1,9 \mathrm{cmol}_{\mathrm{c}} \mathrm{kg}^{-1}$ sem efeito significativo. Esses resultados mostram que a prática de calagem como fator de correçáo do solo favorece o aumento de $\mathrm{Ca}^{2+} \mathrm{e} \mathrm{Mg}^{2+}$ e diminui o teor de $\mathrm{Al}^{3+}$ no substrato. Náo houve efeito da calagem para as demais características.

\section{CRESCIMENTO DAS MUDAS}

Os resultados alcançados demonstraram que as mudas de pau-de-balsa respondem à aplicação de calcário no substrato em altura e diâmetro de colo, com maior desempenho para as mudas que receberam dosagens de corretivo acima de 0,5 t ha $\mathrm{a}^{-1}$ (Tabela 2). Para matéria seca da parte aérea, matéria seca do sistema radicular e matéria seca total, as dosagens de 1,0 a 2,0 tha t $^{-1}$ (Tabela 2) apresentaram melhores resultados, proporcionando os ajustes dos dados a modelos de regressão.

Os modelos de regressão ajustados para as características analisadas das mudas de pau-de-balsa, como altura da planta e diâmetro do colo foram quadrático, porém o desenho sugere modelos lineares (Figura 1). Para a matéria seca da parte aérea, o modelo ajustado foi linear, demonstrando resposta positiva a esta características na proporçấo que se aumenta as doses de calcário aplicadas ao substrato. A matéria seca do sistema radicular e matéria seca total, apresentaram modelos cúbicos (Figura 1).

Tabela 1 - Características químicas dos substratos após 30 dias da aplicação do calcário

\begin{tabular}{|c|c|c|c|c|c|c|c|c|c|c|c|c|}
\hline Dose & $\mathrm{pH}$ & K & $P$ & $\mathrm{Al}^{3+}$ & $\mathrm{Ca}^{2+}$ & $\mathrm{Mg}^{2+}$ & $\mathrm{H}+\mathrm{Al}$ & S & $\mathrm{T}$ & $\mathrm{t}$ & V & $\mathrm{m}$ \\
\hline t ha- $^{-1}$ & $\mathrm{H}_{2} \mathrm{O}$ & \multicolumn{2}{|c|}{-------mg.kg -1------- } & \multicolumn{4}{|c|}{ 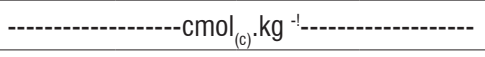 } & \multicolumn{3}{|c|}{ 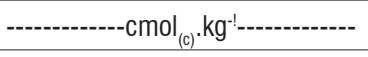 } & \multicolumn{2}{|c|}{------------\%----------- } \\
\hline 0,0 & $3,61 \mathrm{c}$ & $26,8 a b$ & $81,4 a$ & $2,0 \mathrm{a}$ & $0,24 d$ & $0,56 \mathrm{a}$ & $7,4 a b$ & 0,87 & 8,27 & 2,87 & $36,5 c$ & $32,7 \mathrm{a}$ \\
\hline 0,25 & $3,95 \mathrm{e}$ & $26,8 a b$ & $83,6 a$ & $1,7 a b$ & $0,20 \mathrm{~d}$ & $0,78 \mathrm{a}$ & $7,8 \mathrm{a}$ & 1,05 & 8,85 & 2,75 & $38,1 \mathrm{c}$ & $26,7 \mathrm{~b}$ \\
\hline 0,5 & $4,05 \mathrm{de}$ & $21,2 b$ & $87,8 \mathrm{a}$ & $1,6 a b$ & $0,28 d$ & $0,88 a$ & $7,4 a b$ & 1,21 & 8,61 & 2,81 & $40,21 b c$ & $24,3 b$ \\
\hline 0,75 & $4,12 c d$ & $36,0 \mathrm{a}$ & $81,6 a$ & $1,3 b c$ & $0,54 c d$ & $1,0 \mathrm{a}$ & $7,4 a b$ & 1,63 & 9,03 & 2,93 & $41,27 \mathrm{bc}$ & $19,9 \mathrm{bc}$ \\
\hline 1,0 & $4,23 b c$ & $23,2 a b$ & $84,6 a$ & $1,0 \mathrm{c}$ & $0,72 b c$ & $0,96 \mathrm{a}$ & $7,5 a b$ & 1,73 & 9,23 & 2,73 & $41,31 b c$ & $16,2 \mathrm{~cd}$ \\
\hline 1,5 & $4,40 \mathrm{~b}$ & $25,6 a b$ & $85,0 \mathrm{a}$ & $0,9 c$ & $1,02 \mathrm{ab}$ & $1,3 \mathrm{a}$ & $7,1 \mathrm{ab}$ & 2,38 & 9,48 & 3,28 & $44,63 a b$ & $12,9 \mathrm{~cd}$ \\
\hline 2,0 & $4,58 \mathrm{a}$ & $23,4 a b$ & $82,0 \mathrm{a}$ & $0,9 \mathrm{c}$ & $1,22 \mathrm{a}$ & $1,9 a$ & $6,7 \mathrm{~b}$ & 3,17 & 9,87 & 4,07 & $49,02 \mathrm{a}$ & $11,8 \mathrm{~d}$ \\
\hline
\end{tabular}

Médias seguidas pela mesma letra na coluna não diferem entre si pelo teste de Tukey, $\mathrm{P}<0,05$

Tabela 2 - Variáveis mensuráveis das mudas de pau-de-balsa produzidas na casa-de- vegetação.

\begin{tabular}{|c|c|c|c|c|c|c|}
\hline \multirow{2}{*}{ Tratamentos } & Altura & Diâmetro & MSPA & MSR & MST & $\mathrm{R} / \mathrm{PA}$ \\
\hline & (cm) & $(\mathrm{mm})$ & \multicolumn{3}{|c|}{------------------g------------------ } & \\
\hline 0,0 & $29,35 b$ & $7,42 b$ & $8,44 \mathrm{c}$ & $6,94 \mathrm{a}$ & $15,40 b$ & $1,22 \mathrm{a}$ \\
\hline 0,25 & $32,96 b$ & $8,22 b$ & $9,21 b c$ & $8,62 \mathrm{a}$ & $18,00 a b$ & $1,08 \mathrm{a}$ \\
\hline 0,5 & $38,62 a b$ & $8,92 a b$ & $10,23 b c$ & $8,94 \mathrm{a}$ & $19,20 a b$ & $1,16 \mathrm{a}$ \\
\hline 0,75 & $39,77 a b$ & $9,85 a$ & $12,58 a b$ & $9,11 \mathrm{a}$ & $21,70 a b$ & $1,41 \mathrm{a}$ \\
\hline 1,0 & $41,59 a$ & $9,10 a b$ & $12,80 \mathrm{ab}$ & $8,79 \mathrm{a}$ & $21,60 a b$ & $1,52 \mathrm{a}$ \\
\hline 1,5 & $41,09 a$ & $9,32 a b$ & $11,44 a b c$ & $9,36 \mathrm{a}$ & $20,80 a b$ & $1,25 \mathrm{a}$ \\
\hline 2,0 & $43,76 a$ & $9,54 a$ & $13,02 a$ & $10,78 \mathrm{a}$ & $23,80 a$ & $1,22 \mathrm{a}$ \\
\hline
\end{tabular}

Médias seguidas pela mesma letra na coluna não diferem entre si pelo teste de Tukey, $\mathrm{P}<0,05$

MSPA - Matéria seca da parte aérea, MSR - matéria seca da raiz; MST - matéria seca total; R/PA - Relação raiz/Parte aérea 
(a)

Altura

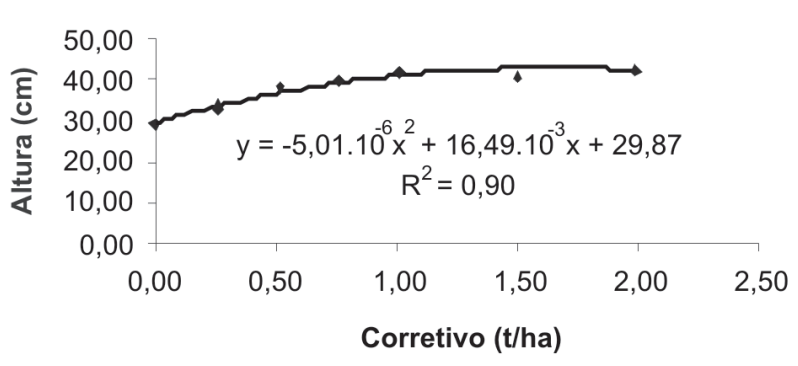

(c)

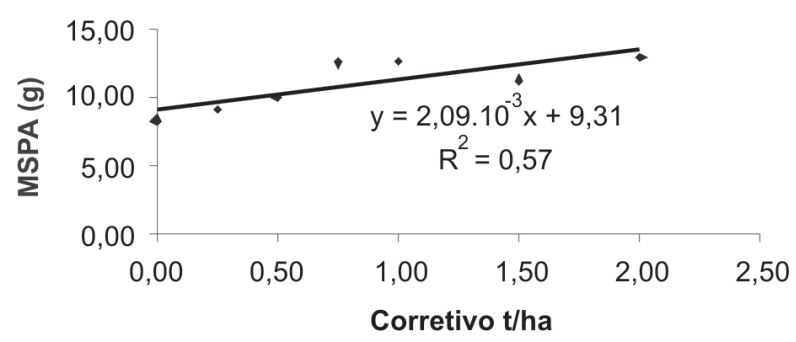

(e)

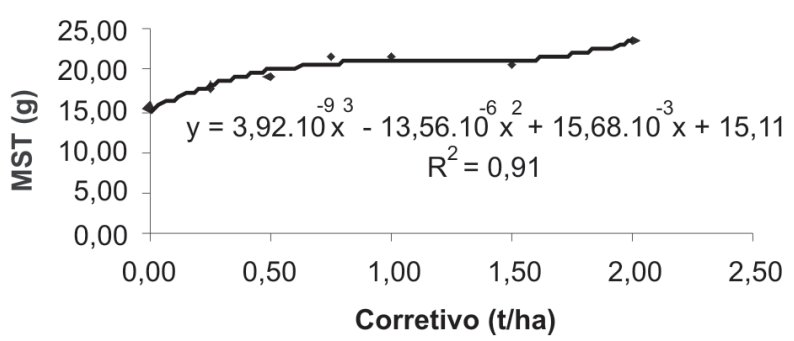

Os melhores desempenhos observados neste estudo podem ser atribuídos ao conteúdo de macronutrientes encontrados nas mudas nos diferentes tratamentos. Resultados semelhantes foram observados por Tucci et al. (2004) e Silva et al. (2008), quando avaliaram o efeito da prática da calagem no substrato sobre a altura, diâmetro de colo e matéria seca de mudas de sumaúma (Ceiba pentandra L. Gaertn). Por outro lado, em estudos realizados por Mann et al. (1996) com acácia (Acácia auriculiforme) e jacarandá-branco (Platipodium elegans) obtiveram resultados diferentes, concluindo que as mudas dessas espécies não responderam a calagem. Conseqüentemente, pode ser considerado que as espécies (b)

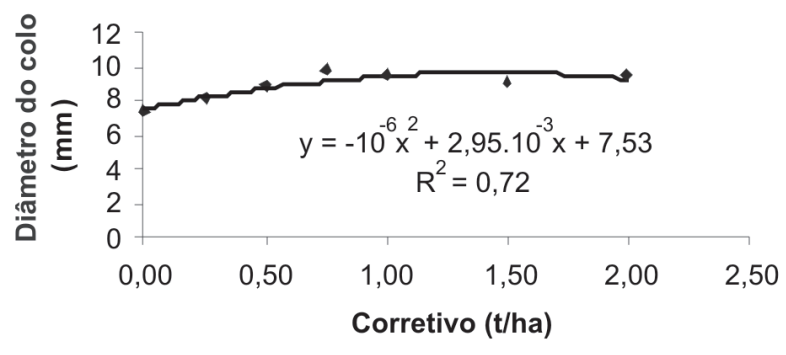

(d)

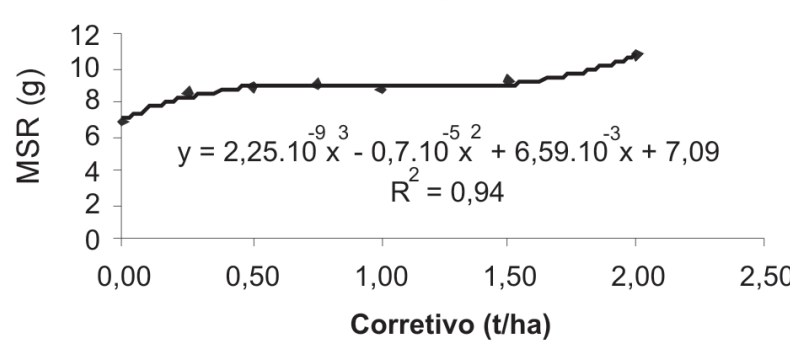

Figura 1 - Equações de regressão ajustadas para as características altura da planta (a), diâmetro do colo (b), matéria seca da parte aérea (c), matéria seca do sistema radicular (d) e matéria seca total (e).

florestais respondem de forma diferenciada à aplicação de calagem.

As diferenças na altura e no diâmetro do colo das mudas com doses de 0,0 a $0,25 \mathrm{t} \mathrm{ha}^{-1}$ foram estatisticamente significativas quando comparadas as doses de 0,5 a $2,0 \mathrm{t} \mathrm{ha}^{-1}$ (Tabela 2). Esses resultados indicam que doses de calcário acima de $0,5 \mathrm{t} \mathrm{ha}^{-1}$, pode contribuir com o aumento no crescimento da altura e no diâmetro do colo das mudas. Os mesmos resultados foram observados por Silva et al. (2008), quando avaliaram o efeito da prática da calagem no substrato sobre a altura e no diâmetro do colo de mudas de sumaúma. Entre as doses de 0,5 a 2,0 t ha ${ }^{-1}$ estatisticamente náo houve 
Tabela 3 - Conteúdo de macronutrientes (N, P, K, Ca, Mg e S) na parte aérea em mudas de pau-de-balsa.

\begin{tabular}{|c|c|c|c|c|c|c|}
\hline \multirow{2}{*}{$\begin{array}{l}\text { Corretivo } \\
\left(\mathrm{t} \mathrm{ha}^{-1}\right)\end{array}$} & $\mathrm{N}$ & $\mathrm{P}$ & K & $\mathrm{Ca}$ & $\mathrm{Mg}$ & $S$ \\
\hline & \multicolumn{6}{|c|}{ 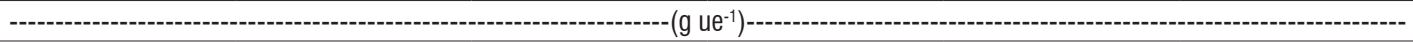 } \\
\hline 0,0 & $23,94 \mathrm{a}$ & $2,23 \mathrm{a}$ & $27,11 \mathrm{a}$ & $8,2 d$ & $1,2 f$ & $1,8 \mathrm{~d}$ \\
\hline 0,25 & $24,56 \mathrm{a}$ & $2,30 \mathrm{a}$ & $23,40 \mathrm{a}$ & $9,6 \mathrm{~d}$ & 3,6 e & $1,9 d$ \\
\hline 0,5 & $27,28 \mathrm{a}$ & $2,33 \mathrm{a}$ & $21,75 \mathrm{a}$ & $11,6 \mathrm{c}$ & $5,1 d$ & $2,3 \mathrm{c}$ \\
\hline 0,75 & $28,36 \mathrm{a}$ & $3,05 \mathrm{a}$ & $25,79 a$ & $15,0 \mathrm{~b}$ & $6,8 \mathrm{c}$ & $2,6 \mathrm{~b}$ \\
\hline 1,0 & $27,76 \mathrm{a}$ & $2,88 \mathrm{a}$ & $24,76 \mathrm{a}$ & $15,4 \mathrm{~b}$ & $7,6 \mathrm{c}$ & $2,7 \mathrm{~b}$ \\
\hline 1,5 & $27,38 \mathrm{a}$ & $2,68 \mathrm{a}$ & $22,94 \mathrm{a}$ & $16,6 \mathrm{~b}$ & $8,3 \mathrm{~b}$ & $3,2 \mathrm{a}$ \\
\hline 2,0 & $27,80 \mathrm{a}$ & $2,94 \mathrm{a}$ & $22,19 \mathrm{a}$ & $20,6 \mathrm{a}$ & $9,4 \mathrm{a}$ & $3,3 \mathrm{a}$ \\
\hline
\end{tabular}

Médias seguidas pela mesma letra na coluna não diferem entre si pelo teste de Tukey, $\mathrm{P}<0,05$

efeito significativos, sendo assim, a dosagem de $0,5 \mathrm{t} \mathrm{ha}^{-1}$ apresentou-se a mais viável economicamente.

Na produção de matéria seca da parte da aérea (MSPA) e matéria seca total (MST), os valores obtidos para as doses de 0,0 a $0,5 \mathrm{t}^{-1} \mathrm{a}^{-1}$ quando comparados com as doses de 0,75 a $2,0 \mathrm{tha}^{-1}$ apresentaram efeito estatisticamente significativos (Tabela 2), evidenciando os efeitos positivos da aplicação de calcário em substrato para a produção de mudas de pau-debalsa. Não se observou efeito da calagem para a relação parte aérea /raiz (Tabela 2).

\section{ABSORÇÃO DE NUTRIENTES}

A calagem em substrato para a produção de mudas de pau-de-balsa apresentou resposta positiva para a absorção de Ca e Mg na presença da dose de 2,0 $\mathrm{t} \mathrm{ha}^{-1}$ (Tabela 3), que pode ser atribuído ao aumento de teores trocáveis desses nutrientes (Silva et al. 2008). Para o $S$ houve resposta nas doses de 1,5 a $2,0 \mathrm{t} \mathrm{ha}^{-1}$. Por outro lado, não foram comprovados efeitos estatisticamente significativos para absorção de $\mathrm{N}, \mathrm{P}$ e $\mathrm{K}$ em nenhuma das doses, evidenciado que a calagem não contribui com o acúmulo desses nutrientes (Tabela 3). Estudos realizados por Silva et al. (2008) com mudas de sumaúma foi comprovado que a calagem favorece a absorção de nitrogênio, fósforo e potássio. Esses resultados, demonstram que as espécies florestais podem responder de forma diferenciada à aplicação de calagem.

Segundo Silva et al. (2008), resultados variados foram encontrados em estudos sobre o efeito da calagem na disponibilidade de fósforo. Tucci et al. (2004) observaram interaçôes positivas entre calagem e adubação fosfatada e negativa para adubação potássica em mudas de sumaúma. Respostas positivas à absorção de potássio têm sido observadas em outros estudos, como observado por Sousa (2000) em açaizeiro (Euterpe oleracea Mart.), a relação cálcio/potássio influenciou nos aspectos nutricionais referentes ao acúmulo de nutrientes na matéria seca da raiz e parte aérea, no acúmulo total e na translocação de nutrientes.

\section{CONCLUSÃO}

A prática de calagem como fator de correção do solo usado no substrato favoreceu todas as características de crescimento avaliadas na produção de mudas de pau-de-balsa, sendo possível o ajuste de equaçóes de regressão.

A correção do solo influenciou positivamente a absorção de $\mathrm{Ca}, \mathrm{Mg}$ e S.

A correção do solo não apresentou efeitos estatisticamente significativos para a absorção de N, P e K.

\section{BIBLIOGRAFIA CITADA}

Barbosa, A.P.; Sampaio, P.T.B.; Campos, M.A.A.; Varela. V.P.; Gonçalves, C.Q.B.; Iida, S. 2004. Alternative technology for breaking dormancy of seeds of pau-de-balsa (Ochroma lagopus Sw, Bombacaceae). Acta Amazônica, 34: 477-482 (in Portuguese, with abstract in English).

Barros, J.G. 2001. Liming and fertilization to the formation of seedlings of mogno (Swietenia macrophylla King). Dissertaçáo de Mestrado, Universidade Federal do Amazonas, Manaus, Amazonas. 64pp (in Portuguese).

Carneiro, J.G.A. 1995. Production and quality control of seedlings of forest species. Curitiba:UFPR/FUPEF. 451 pp (in Portuguese).

Loureiro,A.A.; Silva, M.F.; Alencar, J.C. 1979. Essence lumber Amazon. Vol. II. Instituto Nacional de Pesquisa da Amazônia, Manaus, Amazonas. 187 pp (in Portuguese).

Mann, E.N.; Furtini Neto, A.E.; Resende, A.V.; Vale, F.R.; Fonseca, F.C. Liming and growth of florest species, p. 240-241. 1996. In: Brazilian Meeting of Soil Fertility and Plant Nutrition, 22, Manaus [Palestras...]. Manaus: Ed. da Universidade Federal do Amazonas, Manaus, Amazonas (in Portuguese).

Malavolta, E.; Vitti, G.C.; Oliveira. S.A. 1989. Assessment of nutritional status of plants: principles and applications. Piracicaba: Potafos. 201 pp (in Portuguese).

Ramos, M.B.P.; Varela, V.P.; Melo, M.F.F. 2006. Influence of temperature and the amount of water in the substrate on the germination of Ochroma pyramidale (Cav. Ex Lam) Urban (paude-balsa). Acta Amazônica, 36: 103-106 (in Portuguese, with abstract in English). 
Silva, A.R.M.; Tucci, C.A.F.; Lima, H.N.; Souza, P.A.; Venturin, N. 2008. Effects of increasing doses of lime in the production of seedlings of cedro (Ceiba pentandra L. Gaertn). Revista Floresta. Curitiba, v. 39, n. 2, abril/junho (in Portuguese, with abstract in English).

Sousa, H.U. 2000. Growth and mineral nutrition of seedlings of açai (Euterpe oleraceae Mart.). Tese de Doutorado, Universidade Federal de Lavras, Lavras. 124 pp (in Portuguese).
Tucci, C.A.F.; Hara, F.A.S.; Freitas, R. 2004. Fertilization setting for the formation of the cedro trees (Ceiba pentandra L. Gaertn). Revista de Ciências Agrárias e Ambientais da UFAM, Manaus, v. 11, n. 2/2, jan (in Portuguese, with abstract in English).

Vasquez-Yanes, C. 1974. Studies on the germination of seedes of Ochroma lagopus Sw. Turrialba 24: 176-179 (in English).

Recebido em 12/02/2009

Aceito em 11/12/2009 